\title{
Systematic method of applying ANN for chemical kinetics reduction in turbulent premixed combustion modeling
}

\author{
ZHOU ZhiJun, LÜ Yu, WANG ZhiHua*, XU YanWei, ZHOU JunHu \& CEN KeFa \\ State Key Laboratory of Clean Energy Utilization, Department of Energy Engineering, Zhejiang University, Hangzhou 310027, China
}

Received December 25, 2011; accepted March 9, 2012; published online September 7, 2012

\begin{abstract}
A novel method to apply artificial neural network (ANN) for both chemical kinetics reduction and source term evaluation is introduced and tested in direct numerical simulation (DNS) and large eddy simulation (LES) of reactive flows. To gather turbulence affected flame data for ANN training, a new computation-economical method, called 1D pseudo-velocity disturbed flame (PVDF), is developed and used to generate thermo-chemical states independent of the modeled flame. Then a back-propagation ANN is trained using scaled conjugate gradient algorithm to memorize the sample states with reduced orders. The new method is employed in DNS and LES modeling of $\mathrm{H}_{2} /$ air and $\mathrm{C}_{3} \mathrm{H}_{8}$ /air premixed flames experiencing various levels of turbulence. The test result shows that compared to traditional computation with full mechanism and direct integration, this method can obtain quite large speed-ups with adequate prediction accuracy.
\end{abstract}

ANN, kinetics reduction, LES, DNS

Citation: $\quad$ Zhou Z J, Lü Y, Wang Z H, et al. Systematic method of applying ANN for chemical kinetics reduction in turbulent premixed combustion modeling. Chin Sci Bull, 2013, 58: 486-492, doi: 10.1007/s11434-012-5390-x

Detailed chemical mechanism has been widely employed to numerically study fundamental phenomena involved in flame turbulence interaction, for example, vortex-induced quenching and re-ignition [1], soot [2] and $\mathrm{NO}_{x}$ [3] formation in turbulent flame and flame propagation [4]. However, huge computational burden is always a knotty event during such researches, which mainly stems from two respects: first, large number of species mass balance equations should be temporally advanced; second, many minor species have much smaller time scales than others so that time-consuming stiff ODE solvers are normally required. As a result, complex calculations involved in chemical source evaluation are continually repeated.

In chemical kinetics reduction field, sensitivity analysis [5], quasi-steady state (QSS) assumption [6] and direct relation graphics (DRG) method [7,8] are often used, while ANN method, a extremely new systemic technique, is seldom seen in previous work. The procedure of using QSS assumption has been shown in Figure 1. However, QSS

*Corresponding author (email: wangzh@ zju.edu.cn) species concentrations should be first resolved which includes a large amount of algebraic iterations. Therefore, the net efficiency could be undermined [9], and also calculation may be failed if iterations do not converge.

As for the second respect, several approaches to accelerate chemical sources evaluation have already been presented, such as look-up table (LUT) [10] and in situ-adaptive tabulation (ISAT) [11]. However, they are both based on tabulation technique which needs huge memory and a large number of check-up and interpolation operations. Artificial neural network (ANN) method, although it was proposed previously to handle above drawbacks, has got great progress through Sen and Menon's work [12,13]. It has been successfully applied to account for chemical kinetics in LES modeling of turbulent premixed flame with speed-ups even more than 10 .

In this paper, we tend to extend the application of ANN method, using it to not only calculate chemical sources but also reduce the detailed mechanism. The initial idea is to construct the direct mapping between non-QSS species concentrations and their reaction rates at plenty of thermal 


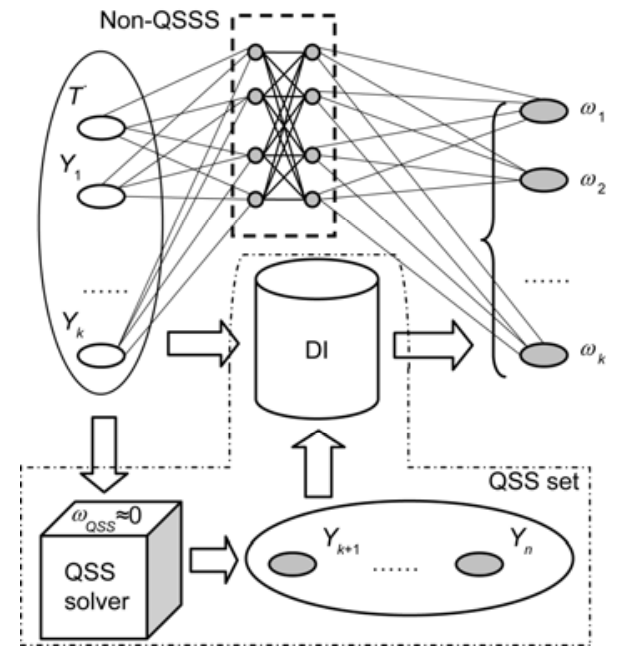

Figure 1 Procedure of using traditional reduced mechanism and ANN-based alternative one (DI means "direct integration").

states considering turbulence effect (see Figure 1). This ANN-based method for chemical kinetics reduction, if demonstrated applicably, can bypass all time-consuming links involved in integration of traditional mechanism and directly offer chemical sources for the mass balance equations of primarily concerned non-QSS species. In other words, it can completely solve the above-mentioned two problems in numerical investigations. Thus, this paper is organized according to the following thread: How is ANNbased method for chemical kinetics reduction constructed? What about the applicability of this method? What is the performance?

\section{Construction of ANN-based method for chemical kinetics reduction}

This ANN-based method is constructed by gathering thermo-chemical sample states, precluding QSS species and employing ANN to memorize the low-order states. All sample states should be at the specific equivalence ratio $\Phi$ with turbulence effect. In [12], sample states were given by DNS of a laminar flame vortex interaction. Although tested performance was satisfactory, this method was very timeconsuming. In this paper, an alternative way with largely reduced calculation is presented. To demonstrate the novel method, premixed combustions of $\mathrm{H}_{2}$ /air at $\Phi=1.0$ and $\mathrm{C}_{3} \mathrm{H}_{8} /$ air at $\Phi=0.65$ are modeled using 11 -species 21 -step [14] and 28-species 73-step [15] mechanisms, respectively. For comparison, no reduction was carried out on $\mathrm{H}_{2} /$ air mechanism, while QSS assumption was imposed on $\mathrm{C}_{3} \mathrm{H}_{8}$ /air combustion process. By adopting the following criterion [6] at laminar $\mathrm{C}_{3} \mathrm{H}_{8} /$ air premixed flame:

$$
\frac{\left|\omega_{s}^{\mathrm{P}}-\omega_{s}^{\mathrm{C}}\right|}{\max \left\{\left|\omega_{s}^{\mathrm{P}}\right|,\left|\omega_{s}^{\mathrm{C}}\right|\right\}} \sim 10^{-2},
$$

where $\omega_{s}$ is chemical reaction rate of specie $s$, superscript $\mathrm{P}$ and $\mathrm{C}$ represent production and consumption, respectively. 7 QSS species are identified, which are $\mathrm{CH}, \mathrm{CH}_{2}, \mathrm{C}_{2} \mathrm{H}_{2}$, $\mathrm{C}_{2} \mathrm{H}_{3}, \mathrm{C}_{2} \mathrm{H}_{5}, \mathrm{C}_{2} \mathrm{H}_{6}$ and $\mathrm{CH}_{2} \mathrm{CO}$. This set of QSS species are eliminated from the gathered sample states. Thus, after being trained, the ANN-based method is actually constructed.

\subsection{Sample data generation}

Sample data for ANN training are initially generated using 1D pseudo-velocity disturbed flame (PVDF) method. The critical idea of this method is to accumulate thermochemical state by stepping following equations with original detailed mechanism:

$$
\begin{gathered}
\rho \frac{\partial\left(Y_{s}\right)}{\partial t}=-\rho u_{t}(x) \frac{\partial\left(Y_{s}\right)}{\partial x}-\frac{\partial\left(\rho Y_{s} V_{s}\right)}{\partial x}-\omega_{s}, \\
c_{v} \rho \frac{\partial T}{\partial t}=-c_{v} \rho u_{t}(x) \frac{\partial(T)}{\partial x}-P \frac{\partial u_{t}(x)}{\partial x}+\frac{\partial}{\partial x}\left(\lambda \frac{\partial T}{\partial x}\right) \\
+\sum_{s} h_{s} \omega_{s}-\frac{\partial T}{\partial x} \rho \sum_{s} Y_{s} V_{s} c_{p s}, \\
\rho=\frac{P}{R_{\mathrm{g}} T}, \quad V_{s}=-D_{s m} \frac{W_{s}}{\bar{W} Y_{s}} \frac{\mathrm{d} X_{s}}{\mathrm{~d} x}, \quad \sum Y_{s} V_{s}=0,
\end{gathered}
$$

where $\rho$ is density, $Y$ is the mass fraction , $u_{t}(x)$ is pseudovelocity used to get disturbed flame data, $V$ is diffusion velocity, $c_{v}$ is mixture specific heat capacity at constant volume, $T$ is temperature, $P$ is pressure, $\lambda$ is thermal conductivity of the mixture, $h$ is standard-state enthalpy, $c_{p}$ is specific heat capacity at constant pressure, $R_{\mathrm{g}}$ is universal gas constant of the mixture, $D_{m}$ is mixture-averaged diffusion coefficient, $X$ is volume fraction, $W$ and $\bar{W}$ are molecular weight and mean molecular weight, respectively. Subscript $s$ represent specie $s$.

1D laminar premixed flame at fixed equivalence ratio (consistent with applied condition) was firstly set to be initial state and then above equations were advanced with 2 th-order difference scheme and 1th-order Euler march. To duplicate turbulence effect on flame, $u_{t}(x)$ is updated at time domain according to an isotropic, homogeneous turbulent kinetic energy spectrum given by [16]

$$
E(k)=\frac{32}{3} \sqrt{\frac{2}{\pi}} \frac{u^{\prime 2}}{k_{e}}\left(\frac{k}{k_{e}}\right)^{4} \exp \left[-2\left(\frac{k}{k_{e}}\right)^{2}\right],
$$

where $k_{e}$ is the most energetic wavenumber in adopted energy spectrum, expressed as

$$
k_{e}=\sqrt{\frac{4 \varepsilon}{10 v u^{\prime 2}}},
$$

$u^{\prime}$ is a preset fluctuation velocity controlling turbulence intensity. To let flame experience different level of turbulence, PVDF was run at 7 different $u^{\prime}$ ranging from $0.5 \mathrm{~m} / \mathrm{s}$ to $u_{\text {max }}^{\prime}$ for both cases. The basic parameters which characterize the turbulent intensity are given in Table 1 . In the following test, $u_{\text {max }}^{\prime}$ is estimated to be $10 \mathrm{~m} / \mathrm{s}$ for $\mathrm{H}_{2} /$ air case and $5 \mathrm{~m} / \mathrm{s}$ for 
Table 1 Parameters of 1D PVDF used to generate training data

\begin{tabular}{ccccc}
\hline$u^{\prime}(\mathrm{m} / \mathrm{s})$ & $R e_{u^{\prime}}$ & Range of $k$ & $k_{e}$ & Time scale $(\mathrm{ms})$ \\
\hline 10 & 5600 & $0-2000$ & 814.8 & 0.261 \\
8 & 4480 & $0-1800$ & 728.8 & 0.365 \\
6 & 3360 & $0-1500$ & 631.2 & 0.562 \\
4 & 2240 & $0-1200$ & 515.3 & 1.032 \\
2 & 1120 & $0-900$ & 364.4 & 2.920 \\
1 & 560 & $0-600$ & 257.7 & 8.260 \\
0.5 & 280 & $0-500$ & 182.2 & 23.361 \\
\hline
\end{tabular}

$\mathrm{C}_{3} \mathrm{H}_{8} /$ air case. In addition, total stepping time for each run was set to be 5 eddy turn-over time $T_{e}$. Required thermochemical states were recorded every $T_{e} / 10$. A hyperplane of PVDF results for $\mathrm{C}_{3} \mathrm{H}_{8}$ /air case, $\mathrm{O}_{2}$ reaction rate along $\mathrm{O}_{2}$ mass fraction (reaction progress), is shown in Figure 2. Turbulent effect on flame is well represented in the observed fluctuations.

\subsection{ANN training}

Back-propagation (BP) network [17] was adopted to memorize the collected sample states. For $\mathrm{H}_{2} /$ air case, QSS hypotheses is not imposed, so all involved species were designated to be inputs; for $\mathrm{C}_{3} \mathrm{H}_{8}$ /air case, because 7 QSS species have been assumed, only remaining 20 species except $\mathrm{N}_{2}$ are needed as inputs and their corresponding chemical rate as outputs. To improve model sensitivity to temperature, sample states were divided into 7 equi-distance bins along temperature value. Further, all data were standardized to range $(0,1)$ before training. Tansig function was used as transfer function. Considering computational efficiency [12], two ANNs are both equipped with two hidden layers. After tentative calculations, ANN of $\mathrm{H}_{2}$ /air case uses 8 neurons for the first hidden layer and 4 for the second; ANN of $\mathrm{C}_{3} \mathrm{H}_{8}$ /air cases is designed with 12 and 6 neurons accordingly. Scaled conjugate gradient (SCG) algorithm first

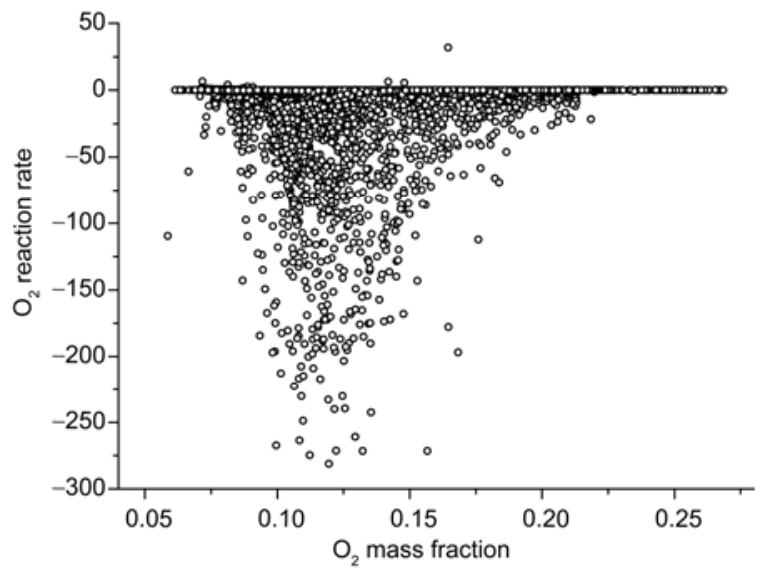

Figure 2 One hyperplane obtained using PVDF approach. proposed by Møller [18] was employed for ANN training. Different from other conjugate gradient algorithm, this algorithm performs no line searches at each iteration. Thus, computation amount and the storage requirement, is largely reduced [19]. The training processes of 7 temperature-data bins for $\mathrm{C}_{3} \mathrm{H}_{8}$ /air mixture are shown in Figure 3. To test ANN performance, 4000 thermo-chemical states are randomly selected from obtained database. Consequently ANN predicted reaction rates perfectly fit the authentic ones, as found in Figure 4. Up to now, ANN-based methods for both flames are constructed and can be directly integrated into following simulations to test the performance.

\section{Numerical methodology for testing}

\subsection{DNS of flame-vortex interaction}

Flame-vortex interaction (FVI) frequently occurs in turbulent combustion, and it represents the basic mode in which premixed flame is affected by vortex [4]. When a vortex penetrates through premixed flame, its leading edge first compresses the encountering flame and then entrainment

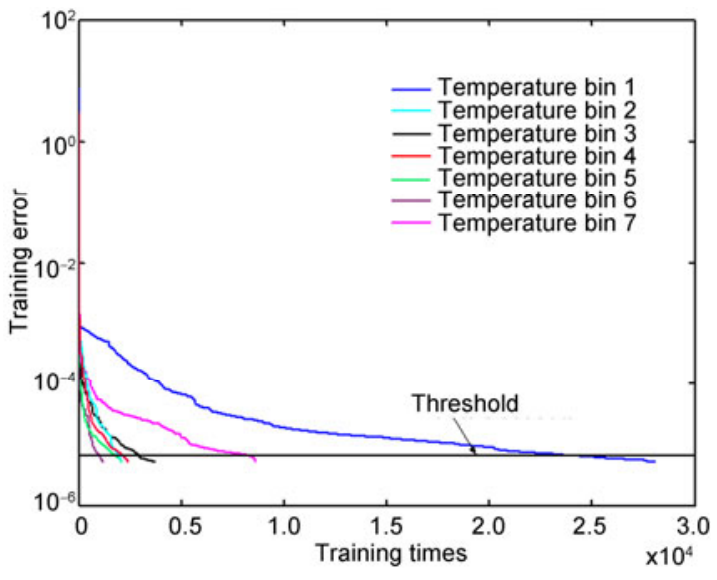

Figure 3 The training processes of all seven temperature-bins for $\mathrm{C}_{3} \mathrm{H}_{8}$ /air mixture.

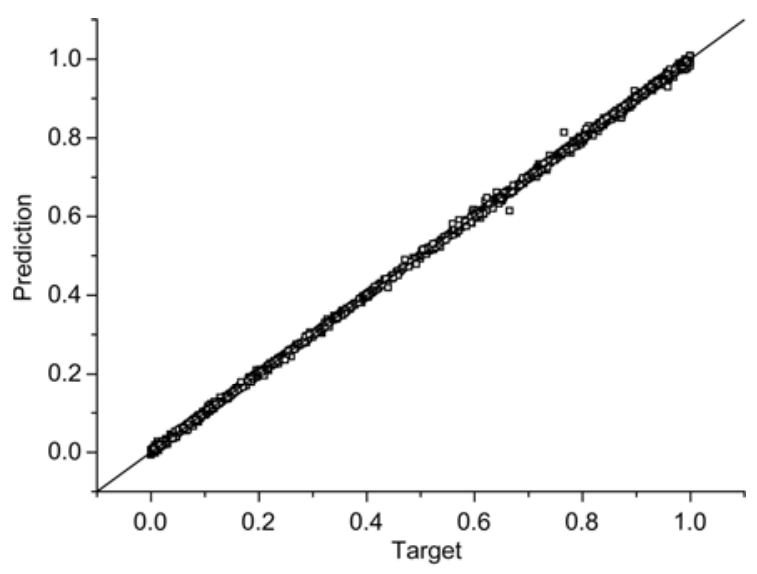

Figure 4 Performance of trained ANN. 
effect causes the adjacent flame being gradually stretched. Consequently, as for the whole flame it experiences dynamically changing strain rate. Whether this process can be accurately modeled by the present method directly indicates its applicability to other models and processes. Thus, our method is designated to be firstly tested in FVI configuration by DNS.

The fully compressible Navier-Stokes equations were firstly nonnormalized, then differenced in eighth-order explicit scheme and then advanced temporarily using the fourth-order explicit Runge-Kutta method with $\mathrm{CFL}=0.06$. Uniform meshes were used with spacing equal to two times of estimated Kolmogolov scale. A modified characteristics non-reflecting boundary condition, which encloses transverse source contribution [20], is imposed on all boundaries. All FVI test cases were initialized using individual laminar premixed flame and a pair of vortex. Laminar premixed flames for both $\mathrm{H}_{2} /$ air and $\mathrm{C}_{3} \mathrm{H}_{8}$ /air cases were obtained by solving PREMIX [14]. The initial vortex pairs were always located in fresh mixture side at $10 l_{f}$ away from the corresponding flame front. For a given moment, scalar profiles were drawn along the trace of one vortex center.

\subsection{LES of turbulent Bunsen flame}

LES of premixed flame has been applied in laboratorial scale [21] and even close to industrial application [22], with the rapid progress of computation ability. Moreover, to satisfy the requirement of modeling auto-ignition and slowscale pollutants production in turbulent premixed flame, it has been believed that reduced mechanism with modest number of species will be initially used. Thus, it is necessary to test the applicability and performance of the present method in LES frame.

In LES, the closure of SGS stresses relies on Boussinesq hypothesis $\tau_{i j}-\tau_{k k} \delta_{i j} / 3=-2 \mu_{\mathrm{t}} \bar{S}_{i j}$, in which $\mu_{\mathrm{t}}$ is the SGS turbulent viscosity, and $\bar{S}_{i j}$ is the rate-of-strain tensor for the resolved scale defined by $\bar{S}_{i j}=\left(\partial \bar{u}_{i} / \partial x_{j}+\partial \bar{u}_{j} /\right.$ $\left.\partial x_{i}\right) / 2$. $\mu_{\mathrm{t}}$ is evaluated using the Dynamic Smagorinsky-
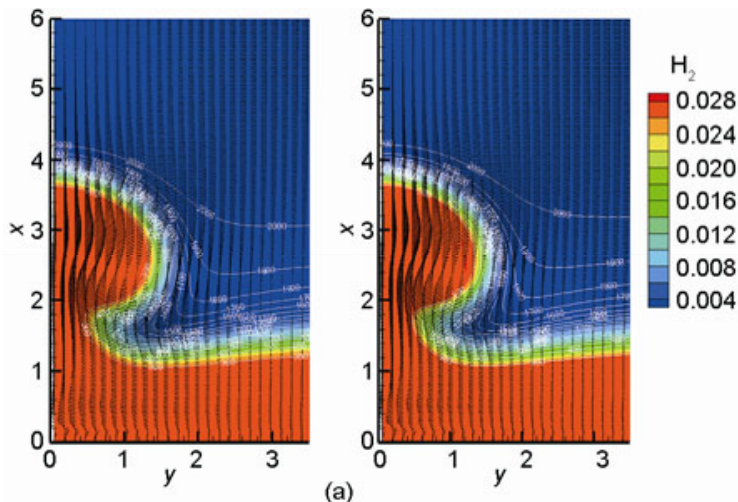

Lilly Model as proposed by Germano et al. [23] and Lilly [24], in which Smagorinsky constant is dynamically computed from local resolved motion to guarantee numerical stability [25]. Eddy-dissipation-concept (EDC) model [26] was adopted to account for combustion in subgrid. Detailed explanation can be found in [21]. For simplicity, LES was implemented in 2D based on finite-volume method. Pressure-velocity coupling was handled according to SIMPLE approach. Spatial differences are 3rd-order bounded center for momentum and second order upwind for others. The second-order accurate time derivative is

$$
\frac{\partial \phi}{\partial t}=\frac{3 \phi^{n+1}-4 \phi^{n}+\phi^{n-1}}{2 \Delta t} .
$$

Integration is implicit; convergence is achieved at each time step in $\sim 20$ iterations. Time step 6e-6s was set with $\mathrm{CFL}=0.1$. For all Bunsen flame cases, velocity, temperature and species concentrations are fixed at inflow boundary, while pressure outlet boundary condition is imposed on all outflow boundaries to ensure "well-posedness" [27]. The components of complementary flow are consistent with those of the corresponding burnt gas at individual adiabatic flame temperature.

\section{Results and discussion}

The performance test focuses on the accuracy, speed-up and storage of the proposed method. Each standard solution was obtained using the corresponding original detailed mechanisms and direct integration.

\subsection{FVI test}

Different vortex pairs were used in FVI test. $\mathrm{H}_{2} /$ air FVI cases were run with $U_{\max } / S_{L}$ equal to 26 and 39 while $\mathrm{C}_{3} \mathrm{H}_{8} /$ air FVI cases were obtained at 33 and $66 U_{\max } / S_{L}$ conditions. $U_{\max }$ is the maximum velocity induced by vortex. $2 \mathrm{D}$ contours with velocity vector plots, predicted using two methods, are shown in Figure 5. Furthermore, instantaneous profiles of scalars cross the individual flame are summarized in Figure 6.
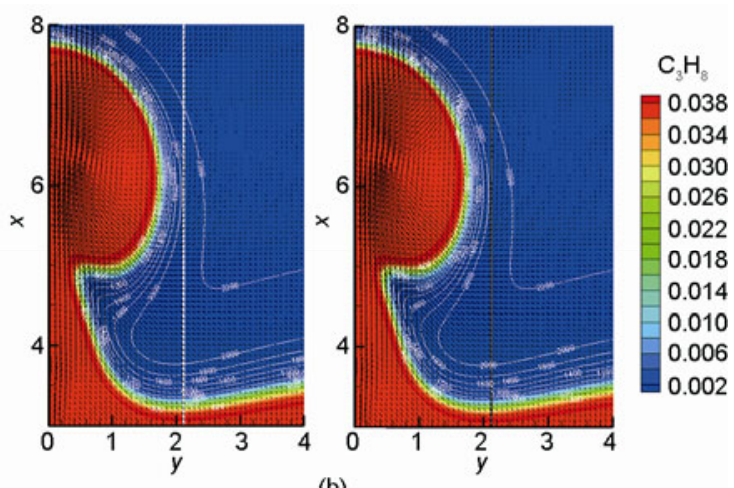

(b)

Figure 5 2D contours with velocity vectors predicted using DI and ANN for $\mathrm{H}_{2} /$ air FVI with $U_{\max } / S_{L}=26$ (a) and $\mathrm{C}_{3} \mathrm{H}_{8} /$ air FVI with $U_{\max } / S_{L}=66$ (b): DI (left); ANN represented method (right). Colored by corresponding species mass fraction and lined by temperature value. 

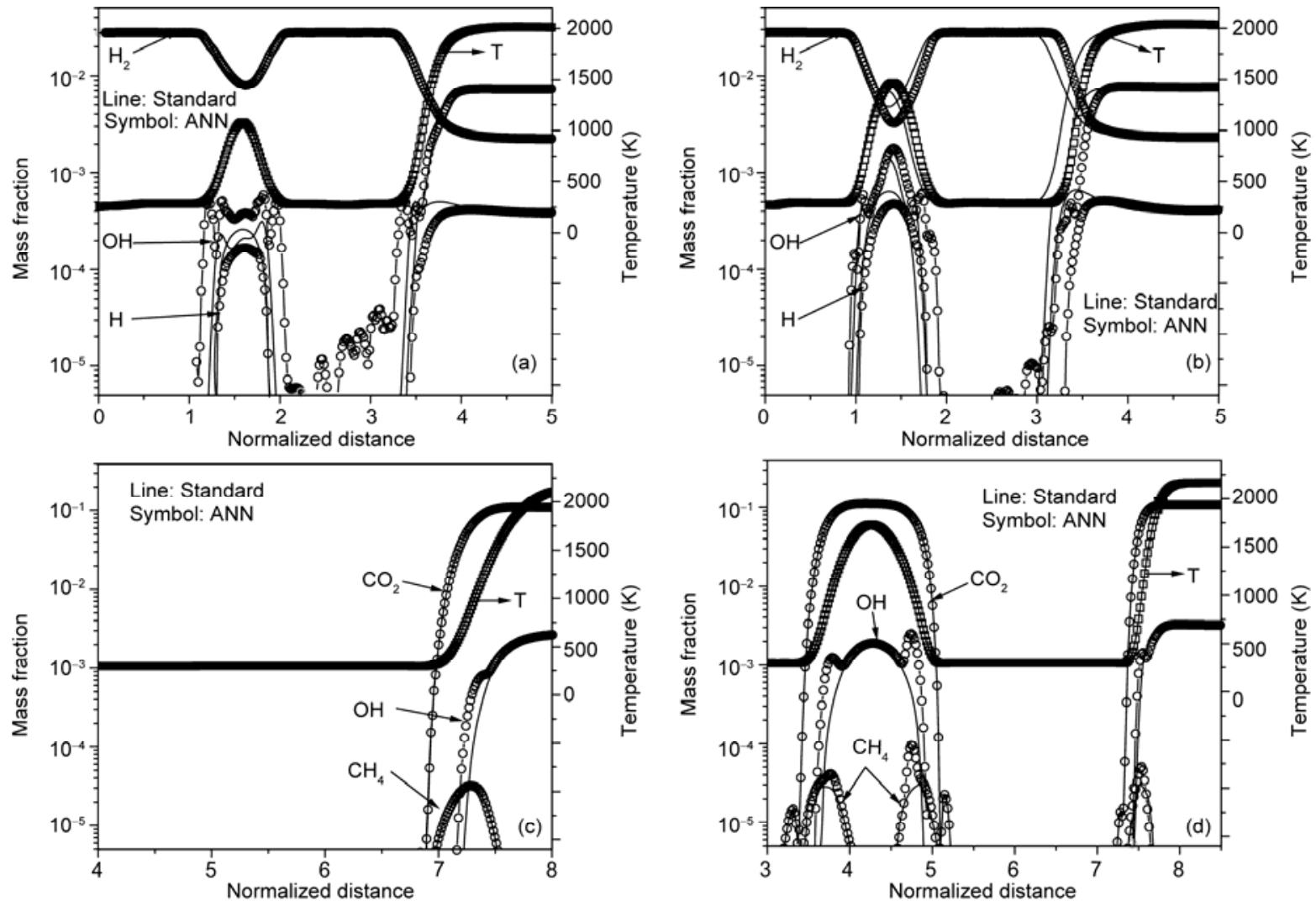

Figure 6 Instantaneous profiles of scalar variables in DNS of FVI. $\mathrm{H}_{2} /$ air case: (a) $U_{\max } / S_{L}=26$; (b) $U_{\max } / S_{L}=39 ; \mathrm{C}_{3} \mathrm{H}_{8} /$ air case: (c) $U_{\max } / S_{L}=33$; (d) $U_{\max } / S_{L}=66$

$\mathrm{H}_{2} /$ air FVI cases are shown in Figure 6(a) and (b) while $\mathrm{C}_{3} \mathrm{H}_{8} /$ air FVI cases are in Figure 6(c) and (d). All predicted profiles well match with the corresponding standard ones. At all observed extremums, the relative errors of major species are not larger than $23 \%$. On average errors are below $5 \%$ for major species and temperature. For minor species, such as $\mathrm{OH}, \mathrm{H}$ and $\mathrm{CH}_{4}$, errors are a little larger though profile agreements are still preserved. Predicted profiles of other involved species represent similar features even if not shown in figure. Comparatively scalar errors are a little larger in $\mathrm{C}_{3} \mathrm{H}_{8} /$ air case than those in $\mathrm{H}_{2}$ /air case due to inclusion of kinetics reduction process, but by referring to the errors resulting from traditional reduced mechanism [9,28-31], the error magnitude is quite acceptable. Overall, the ANN-based method for chemical kinetics reduction can well replicate the flame dynamics in FVI at the influence of different vortex.

\subsection{Bunsen-flame test}

The instantaneous flame structures of the tested cases are shown in Figure 7. The $\mathrm{H}_{2} /$ air Bunsen flame uses fresh mixture inflow at $298 \mathrm{~K}$ with $1.5 \times 10^{5}$ Reynolds number. The size of the jet is $70 \mathrm{~mm}$, with the speed of $200 \mathrm{~m} / \mathrm{s}$ centerflow and $1.8 \mathrm{~m} / \mathrm{s}$ coflow. The inflow property of
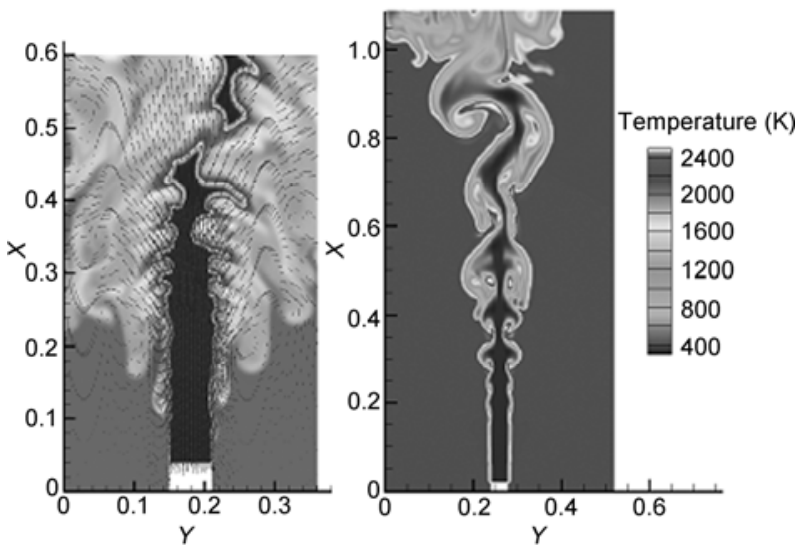

Figure 7 Instantaneous temperature fields of Bunsen-flame LES using ANN-based method for chemical kinetics reduction. (a) $\mathrm{H}_{2} /$ air case; (b) $\mathrm{C}_{3} \mathrm{H}_{8}$ /air case.

$\mathrm{C}_{3} \mathrm{H}_{8} /$ air flame is $6.2 \times 10^{4}$ Reynolds number at $298 \mathrm{~K}$. The size of the jet is $32 \mathrm{~mm}$, with the speed of $8 \mathrm{~m} / \mathrm{s}$ centerflow and $0.4 \mathrm{~m} / \mathrm{s}$ coflow. Both grids are $L_{x} \times L_{y}=3 \mathrm{~mm} \times 3 \mathrm{~mm}$, with stretch ratio of 1.05 in PML areas. As seen, along streamwise flame thickness is gradually increased and wrinkles become more obvious owing to vortex stretch effect. Comparatively $\mathrm{C}_{3} \mathrm{H}_{8} /$ air flame is stretched to a more serious extent because of its much lower flame velocity. 
Quantified accuracy evaluation is carried out by comparing ANN-based method predictions with the corresponding standard ones. Detailed comparisons of Favre-averaged scalar profiles have been exhibited in Figure 8. The predicted profiles are all in good agreement with standard solutions. Further, the relative errors of major species and temperature are around $13 \%$ and those of minor species are approximately $22 \%$. Further, it is noticeable that the change of flame thicknesses and flame positions along streamwise is well resolved using the present method, especially in $\mathrm{C}_{3} \mathrm{H}_{8}$ /air case. However, in some locations errors are larger for some minor species, such as $\mathrm{C}_{2} \mathrm{H}_{4}$ and $\mathrm{O}$. The reason is probably the insensitivity of trained ANN to the used chemical sub-model in LES [32], which offers a good starting point for further improvement.

\subsection{Speed-up and storage summary}

For each case, the speed-up is defined as the ratio of running time using full mechanism with ANN-based method in each CFD time step. The running time used here actually include both time consumed for chemical reaction and fluid dynamics. As listed in Table 2, speed-ups obtained in DNS are relatively low. However, considering DNS usually run for many days or even several months, the absolute time actually saved is considerable. In LES, 13.6 speed-up obtained here is also larger than Sen and Menon's optimal value 11.2 in [12]. In addition, the required storage of our

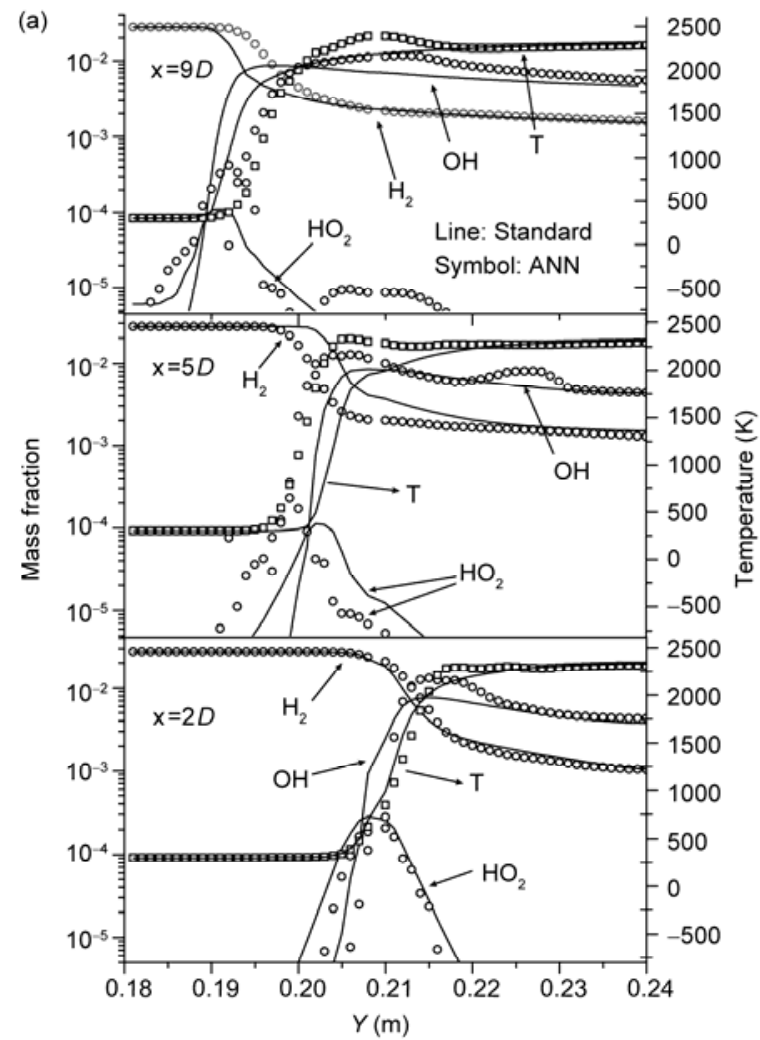

method is very negligible, and thus it is still superior to other tabulation technics.

\section{Conclusions}

The construction and application of a novel ANN-based method for chemical kinetics reduction is discussed in detail. The PVDF approach is involved to quickly generate thermo-chemical states for ANN training. Pseudo-velocity, which can lead premixed flame to experiencing turbulence with various time and length scales, is introduced. The adaptability to different chemical kinetics, prediction accuracy, computational efficiency and storage of the method are highlighted in the performance test. $\mathrm{H}_{2}$ /air and $\mathrm{C}_{3} \mathrm{H}_{8}$ /air turbulent premixed flames are modeled by DNS and LES integrated with this ANN-based method. The result shows that compared with ODE solutions using original detailed mechanism, the present method can well predict scalar profiles and error magnitudes of species concentrations and temperature are acceptable. Moreover, due to the involved

Table 2 Characteristic parameters of ANN-based method for both cases

\begin{tabular}{cccccc}
\hline Case & $\begin{array}{c}\text { Kinetics } \\
\text { size }\end{array}$ & $\begin{array}{c}\text { Table } \\
\text { storage }\end{array}$ & $\begin{array}{c}\text { ANN } \\
\text { storage }\end{array}$ & $\begin{array}{c}\text { Speed-up } \\
\text { in DNS }\end{array}$ & $\begin{array}{c}\text { Speed-up in } \\
\text { LES }\end{array}$ \\
\hline $\mathrm{H}_{2} /$ air & 11 species & $160 \mathrm{Mb}$ & $175 \mathrm{~kb}$ & 1.05 & 8.2 \\
$\mathrm{C}_{3} \mathrm{H}_{8} /$ air & 20 species & $330 \mathrm{Mb}$ & $432 \mathrm{~kb}$ & 1.42 & 13.6 \\
\hline
\end{tabular}

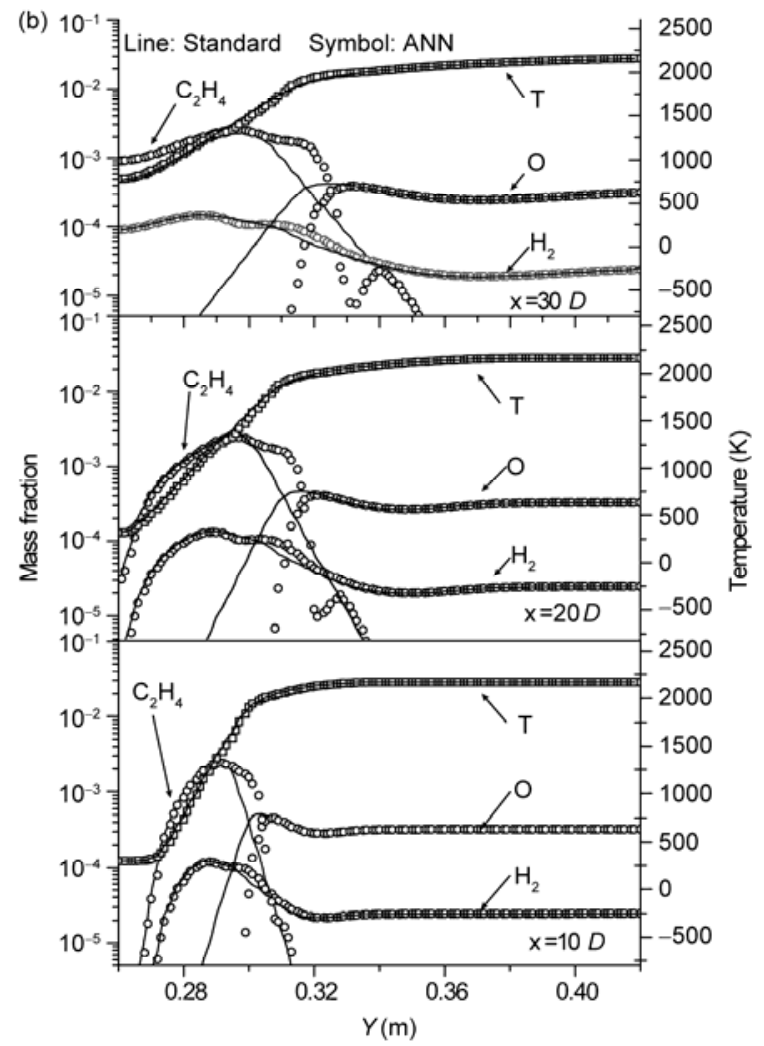

Figure 8 Favre-averaged scalar profiles at different cross-sections downstream. (a) $\mathrm{H}_{2} /$ air case; (b) $\mathrm{C}_{3} \mathrm{H}_{8} /$ air case. 
mechanism reduction, $\mathrm{C}_{3} \mathrm{H}_{8} /$ air case obtains a higher speedup in LES than $\mathrm{H}_{2} /$ air case and previous study. The required storages of the present method for both cases are quite negligible (below $500 \mathrm{~kb}$ ), much smaller than LUT and ISAT techniques. Thus, this method is more advantageous in memory-limited computations.

This work was supported by the National Natural Science Foundation of China (51176169) and the Program of Introducing Talents of Discipline to University (B08026). The authors also would like to thank Professor Haworth from the Pennsylvania State University for providing propane combustion mechanism.

1 Venugopal R, Abraham J. A 2-D DNS investigation of extinction and reignition dynamics in nonpremixed flame-vortex interactions. Combust Flame, 2008, 153: 442-464

2 Lignell D O, Chen J H, Smith P J, et al. The effect of flame structure on soot formation and transport in turbulent nonpremixed flames using direct numerical simulation. Combust Flame, 2007, 151: 2-28

3 Xue H, Aggarwal S K. NO emissions in n-heptane/air partially premixed flames. Combust Flame, 2003, 132: 723-741

4 Kolera-Gokula H, Echekki T. Direct numerical simulation of premixed flame kernel-vortex interactions in hydrogen-air mixtures. Combust Flame, 2006, 146: 155-167

5 Tomlin A S, Turanyi T, Pilling M J. Comprehensive Chemical Kinetics. Amsterdam: Elsevier, 1997. 293-437

6 Lv Y, Wang Z, Zhou J, et al. Reduced mechanism for hybrid $\mathrm{NO}_{x}$ control process. Energy Fuels, 2009, 23: 5920-5928

7 Lu T, Law C K. A directed relation graph method for mechanism reduction. Proc Combust Inst, 2005, 30: 1333-1341

$8 \mathrm{Lu} \mathrm{T}$, Law C K. Linear time reduction of large kinetic mechanisms with directed relation graph: n-heptane and iso-octane. Combust Flame, 2006, 144: 24-36

9 Lv Y, Wang Z, Zhou J, et al. Development and validation of a reduced mechanism for urea-based SNCR process based on QSS graph. Energy Fuels, 2009, 23: 3605-3611

10 Chen J Y, Kollmann W, Dibble R W. Pdf modeling of turbulent nonpremixed methane jet flames. Combust Sci Tech, 1989, 64: 315-346

11 Pope S B. Computationally efficient implementation of combustion chemistry using in situ adaptive tabulation. Combust Theor Model, 1997, 1: 41-63

12 Sen B A, Menon S. Turbulent premixed flame modeling using artificial neural networks based chemical kinetics. Proc Combust Inst, 2009, 32: 1605-1611

13 Sen B A, Menon S. Artificial neural networks based chemistry-mixing subgrid model for LES. AIAA-07-0241, 47th Aerospace
Science Meeting and Ex-hibit, 2007

14 Kee R J, Rupley F M, Miller J A. Chemkin-II: A fortran chemical kinetics package for the analysis of gas-phase chemical kinetics. Report No. SAND89-8009, Sandia National Laboratories, 1989

15 Haworth D C, Blint R J, Cuenot B, et al. Numerical simulation of turbulent propane-air combustion with nonhomogeneous reactants. Combust Flame, 2000, 121: 395-417

16 Passot T, Pouquet A. Numerical simulation of compressible homogeneous flows in the turbulent regime. J Fluid Mech, 1987, 181: 441-466

17 Rumelhart D E, Hinton G E, Williams R J. Learning representations by back-propagating errors. Nature, 1986, 323: 533-536

18 Møller M F. A scaled conjugate gradient algorithm for fast supervised learning. Neural Networks, 1993, 6: 525-533

19 Plumb A P, Rowe R C, York P, et al. Optimisation of the predictive ability of artificial neural network (ANN) models: A comparison of three ANN programs and four classes of training algorithm. Europ J Pharm Sci, 2005, 25: 395-405

20 Lodato G, Domingo P, Vervisch L. Three-dimensional boundary conditions for direct and large-eddy simulation of compressible viscous flows. J Comput Phys, 2008, 227: 5105-5143

21 Giacomazzi E, Battaglia V, Bruno C. The coupling of turbulence and chemistry in a premixed bluff-body flame as studied by LES. Combust Flame, 2004, 138: 320-335

22 Boileau M, Staffelbach G, Cuenot B, et al. LES of an ignition sequence in a gas turbine engine. Combust Flame, 2008, 154: 2-22

23 Germano M, Piomelli U, Moin P, et al. A dynamic subgrid-scale eddy viscosity model. Phys Fluids A: Fluid Dyn, 1991, 3: 1760-1765

24 Lilly D K. A proposed modification of the Germano subgrid-scale closure method. Phys Fluids A: Fluid Dyn, 1992, 4: 633-635

25 Kim S E. Large eddy simulation using unstructured meshes and dynamic subgrid-scale turbulence models. AIAA-2004-2548, 34th Fluid Dynamics Conference and Exhibit, 2004

26 Magnussen B F, Hjertager B H. On mathematical modeling of turbulent combustion with special emphasis on soot formation and combustion. Int Symp Combust, 1977, 16: 719-729

27 Poinsot T J, Lelef S K. Boundary conditions for direct simulations of compressible viscous flows. J Comput Phys, 1992, 101: 104-129

28 Giral I, Alzueta M U. An augmented reduced mechanism for the reburning process. Fuel, 2002, 81: 2263-2275

29 Mendiara T, Alzueta M U, Millera A, et al. An augmented reduced mechanism for methane combustion. Energy Fuels, 2004, 18: 619-627

$30 \mathrm{Xu} \mathrm{H}$, Smoot L D, Hill S C. A reduced kinetic model for $\mathrm{NO}_{x}$ reduction by advanced reburning. Energy Fuels, 1998, 12: 1278-1289

$31 \mathrm{Xu} \mathrm{H}$, Smoot L D, Hill S C. Computational model for $\mathrm{NO}_{x}$ reduction by advanced reburning. Energy Fuels, 1999, 13: 411-420

32 Sen B A, Menon S. Linear eddy mixing based tabulation and artificial neural networks for large eddy sim-ulations of turbulent flames. Combust Flame, 2010, 157: 62-74

Open Access This article is distributed under the terms of the Creative Commons Attribution License which permits any use, distribution, and reproduction in any medium, provided the original author(s) and source are credited. 